\title{
Existence Results for Fractional Semilinear Integrodifferential Equations of Mixed Type with Delay
}

\author{
Xue Wang $\mathbb{D}^{1,2}$ and Bo Zhu $\mathbb{D}^{2}$ \\ ${ }^{1}$ School of Mathematics, Shandong University, Jinan 250100, China \\ ${ }^{2}$ School of Mathematics and Quantitative Economics, Shandong University of Finance and Economics, Jinan 250014, China \\ Correspondence should be addressed to Bo Zhu; zhubo207@163.com
}

Received 18 January 2021; Revised 9 February 2021; Accepted 23 February 2021; Published 2 March 2021

Academic Editor: Chuanjun Chen

Copyright (c) 2021 Xue Wang and Bo Zhu. This is an open access article distributed under the Creative Commons Attribution License, which permits unrestricted use, distribution, and reproduction in any medium, provided the original work is properly cited.

In this paper, we discuss a class of fractional semilinear integrodifferential equations of mixed type with delay. Based on the theories of resolvent operators, the measure of noncompactness, and the fixed point theorems, we establish the existence and uniqueness of global mild solutions for the equations. An example is provided to illustrate the application of our main results.

\section{Introduction}

Fractional calculus can be used to describe some nonclassical phenomena in natural science and engineering applications. Fractional differential equations have been applied in different fields ranging from engineering, finance, and physics in the past few decades. Researchers have conducted extensive explorations on this subject and have achieved fruitful results for the fractional differential equations [1-13]. Zhu and Han [10] and Chadha and Pandey [11] studied the fractional integrodifferential equations and discussed the existence of mild solutions. Based on the theory of the resolvent family and fixed point theorems, Chen et al. [14-17] analyzed nonautonomous evolution equations in a Banach space. Moreover, some researchers considered sufficient conditions on the existence of mild solutions for fractional differential equations by the measure of noncompactness $[4,18,19]$. The initial boundary value problem for the fractional integrodifferential equations with delay has been investigated by using fixed point theorems $[4,5,18,20]$. In [3, 21-24], differential equations of mixed type have been studied and some results have been concluded.

Chen [22] studied the fractional nonautonomous evolution equations of mixed type:

$$
\left\{\begin{array}{l}
{ }^{c} D_{t}^{\beta} u(t)+A(t) u(t)=f(t, u(t), T u(t), S u(t)), \quad t \in(0, a], \\
u(0)=A^{-1}(0) u_{0},
\end{array}\right.
$$

where

$$
\begin{aligned}
& T u(t)=\int_{0}^{t} K(t, s) u(s) d s, \\
& S u(t)=\int_{0}^{a} H(t, s) u(s) d s,
\end{aligned}
$$

where the kernels $K$ and $H$ are linear functions. The operator $T$ is an integral with a variable upper limit, and the operator $S$ is an ordinary definite integral; accordingly, problem (1) is called fractional semilinear integrodifferential equations of mixed type.

Li and Jia [25] investigated the existence of mild solutions for abstract delay fractional differential equations:

$$
\left\{\begin{array}{l}
{ }^{c} D_{t}^{\beta} u(t)=A u(t)+J_{t}^{1-\beta} f\left(t, u_{t}\right), \quad t \in[0, T], \\
u(t)=\varphi(t), \quad t \in[-r, 0],
\end{array}\right.
$$


where $\beta \in(0,1), J_{t}^{1-\beta}$ is the Riemann-Liouville fractional integral, the linear operator $A$ is independent on $t$, and the Lipschitz coefficient of $f$ is constant.

To the best of our knowledge, there are no results on the fractional integrodifferential equations of mixed type with delay. Motivated by this idea, we consider the following problem:

$$
\left\{\begin{array}{l}
{ }^{c} D_{t}^{\beta} x(t)=A(t) x(t)+J_{t}^{1-\beta} f\left(t, x_{t}, \mathscr{K} x_{t}, \mathscr{H} x_{t}\right), \quad t \in\left[0, T_{0}\right], \\
x(t)=\phi(t), \quad t \in[-r, 0],
\end{array}\right.
$$

where $\beta \in(0,1],{ }^{c} D_{t}^{\beta}$ is the Caputo fractional derivative of order $\beta, A(t)$ is a closed and linear operator with domain $D(A)$ defined on a Banach space $E, J_{t}^{1-\beta}$ is the RiemannLiouville fractional integral of order $1-\beta, \mathscr{K}$ and $\mathscr{H}$ are defined by

$$
\begin{aligned}
\mathscr{K} x_{t} & =\int_{0}^{t} K\left(t, s, x_{s}\right) d s, \\
\mathscr{H} x_{t} & =\int_{0}^{T_{0}} H\left(t, s, x_{s}\right) d s,
\end{aligned}
$$

where $K: D \times C([-r, 0] ; E) \longrightarrow E$ and $H: D_{0} \times C([-r, 0] ; E)$ $\longrightarrow E$ are continuous and nonlinear functions, $D=\{(t, s)$ $\left.\in R^{2}: 0 \leq s \leq t \leq T_{0}\right\}, J=\left[0, T_{0}\right], D_{0}=\left\{(t, s) \in R^{2}: 0 \leq t, s \leq\right.$ $\left.T_{0}\right\}, \phi \in C[-r, 0], f$ is to be specified later, and $x_{t}$ means the element of $C([-r, 0] ; E)$ defined by $x_{t}(\theta)=x(t+\theta),-r$ $\leq \theta \leq 0$, for $x \in C\left(\left[-r, T_{0}\right] ; E\right), t \in J$.

We demonstrate the existence and uniqueness of global mild solutions for problem (4) under the conditions of the compact resolvent operator and noncompact resolvent operator, respectively. The kernels $K$ and $H$ of the operators $\mathscr{K}$ and $\mathscr{H}$ are nonlinear functions. In addition, the operator $A$ $(t)$ is dependent on $t$. The rest of this paper is organized as follows. Basic definitions and auxiliary results are presented in Section 2. In Section 3, we prove the existence and uniqueness of mild solutions via various fixed point theorems, the measure of noncompactness, and the Banach contraction mapping principle. An example is provided to illustrate the main theorems in Section 4. Finally, Section 5 is the summary of our results.

\section{Preliminaries}

Definition $1[6,26]$. The Riemann-Liouville fractional integral $J_{t}^{\beta}$ and derivative $D_{t}^{\beta}$ of a function $f:(0, \infty) \longrightarrow R$ of order $\beta>0$ are defined by

$$
\begin{aligned}
J_{t}^{\beta} f(t) & =\frac{1}{\Gamma(\beta)} \int_{0}^{t}(t-s)^{\beta-1} f(s) d s, \\
D_{t}^{\beta} f(t) & =\frac{1}{\Gamma(n-\beta)}\left(\frac{d}{d t}\right)^{n} \int_{0}^{t}(t-s)^{n-\beta-1} f(s) d s, \quad n-1<\beta \leq n,
\end{aligned}
$$

where $f(t) \in L^{1}\left(\left(0, T_{0}\right) ; E\right), \Gamma(\cdot)$ denotes the gamma function, and $n \in N$.

Remark 2 [25]. $D_{t}^{\beta} f(t)=D_{t}^{m} J_{t}^{m-\beta} f(t)$, where $D_{t}^{m}=d^{m} / d t^{m}$ and $J_{t}^{m-\beta} f(t) \in W^{m, 1}\left(\left(0, T_{0}\right) ; E\right)$.

Definition 3 [26, 27]. The Caputo fractional derivative of order $\beta>0$ of a function $f:(0, \infty) \longrightarrow R$ is given by

$$
{ }^{c} D_{t}^{\beta} f(t)=\frac{1}{\Gamma(n-\beta)} \int_{0}^{t}(t-s)^{n-\beta-1} f^{(n)}(s) d s, \quad n-1<\beta<n .
$$

Remark 4 [25]. For the Riemann-Liouville fractional integral operator and the Caputo fractional derivative operator, the following conclusions are obtained:

$$
\begin{aligned}
{ }^{c} D_{t}^{\beta} f(t) & =D_{t}^{\beta}\left(f(t)-\sum_{k=0}^{m-1} \frac{t^{k}}{k !} f^{(k)}(0)\right), \\
{ }^{c} D_{t}^{\beta}\left(J_{t}^{\beta} f(t)\right) & =f(t), \\
J_{t}^{\beta}\left({ }^{c} D_{t}^{\beta}(f(t))\right) & =f(t)-\sum_{k=0}^{m-1} \frac{t^{k}}{k !} f^{(k)}(0) .
\end{aligned}
$$

Definition $5[28,29]$. Let $A(t)$ be a closed and linear operator with domain $D(A)$ defined on a Banach space $E$ and $\beta>0$. Let $\rho[A(t)]$ be the resolvent set of $A(t) . A(t)$ is called the generator of a $\beta$-resolvent family if there exist $\omega \geq 0$ and a strongly continuous function $U_{\beta}: R_{+}^{2} \longrightarrow B(E)$ such that $\left\{\lambda^{\beta}: \operatorname{Re} \lambda>\omega\right\} \subset \rho(A)$ and

$$
\left(\lambda^{\beta} I-A(s)\right)^{-1} x=\int_{0}^{\infty} e^{-\lambda(t-s)} U_{\beta}(t, s) x d t, \quad \operatorname{Re}(\lambda)>\omega, x \in E .
$$

In this case, $U_{\beta}(t, s)$ is called the $\beta$-resolvent family generated by $A(t)$.

Remark $6[29,30] . U_{\beta}(t, s)$ satisfies the following properties:

(1) $U_{\beta}(s, s)=I$ and $U_{\beta}(t, s)=U_{\beta}(t, r) U_{\beta}(r, s)$, for $0 \leq s$ $\leq r \leq t \leq a$

(2) $(t, s) \longrightarrow U_{\beta}(t, s)$ is strongly continuous for $0 \leq s \leq t$ $\leq a$

(3) If $U_{\beta}(t, s)$ is compact for $t, s>0$, then $U_{\beta}(t, s)$ is continuous in the uniform operator topology

Lemma 7 [21]. Let $B \subset C[J, E]$ be equicontinuous and bounded; then, $\bar{C} o B \subset C[J, E]$ is also equicontinuous and bounded. 
Lemma 8 [24]. Let $B \subset C[J, E]$ be equicontinuous and bounded; then, $\alpha(B(t))$ is continuous on J and

$$
\alpha\left(\int_{J} B(s) d s\right) \leq \int_{J} \alpha(B(s) d s), \quad \alpha(B)=\max _{t \in J} \alpha(B(t)),
$$

where $\alpha$ denotes the measure of noncompactness.

Lemma 9 [21]. Let $E$ be a Banach space and $D \subset E$ be bounded; then, there exists a countable set $D_{0} \subset D$ such that $\alpha(D) \leq 2 \alpha\left(D_{0}\right)$.

Lemma 10 [31]. Let $E$ be a Banach space and $D \subset E$ be a bounded closed and convex set. Assume that $Q: D \longrightarrow D$ is a strict set contraction mapping; then, $Q$ has at least one fixed point in $D$.

Definition 11. A function $x \in C\left(\left[-r, T_{0}\right] ; E\right)$ is a mild solution of problem (4), if $x$ satisfies the following equations:

$$
x(t)=\left\{\begin{array}{l}
U_{\beta}(t, 0) \phi(0)+\int_{0}^{t} U_{\beta}(t, s) f\left(s, x_{s}, \mathscr{K} x_{s}, \mathscr{H} x_{s}\right) d s, \quad t \in\left[0, T_{0}\right], \\
\phi(t), \quad t \in[-r, 0] .
\end{array}\right.
$$

\section{Main Results}

Let us introduce the operator $\Psi: C\left(\left[-r, T_{0}\right] ; E\right) \longrightarrow C([-r$, $\left.\left.T_{0}\right] ; E\right)$ by

$$
\Psi x(t)=\left\{\begin{array}{l}
U_{\beta}(t, 0) \phi(0)+\int_{0}^{t} U_{\beta}(t, s) f\left(s, x_{s}, \mathscr{K} x_{s}, \mathscr{H} x_{s}\right) d s, \quad t \in\left[0, T_{0}\right], \\
\phi(t), \quad t \in[-r, 0] .
\end{array}\right.
$$

Theorem 12. Assume that the following conditions hold:

$\left(H_{1}\right)$. The resolvent operator $U_{\beta}(t, s)$ is compact for all $t$, $s>0, M^{\star}=\max \left\|U_{\beta}(t, s)\right\|<+\infty, 0 \leq s \leq t \leq T_{0}$.

$\left(H_{2}\right) . K: D \times C([-r, 0] ; E) \longrightarrow E$ and $H: D_{0} \times C([-r, 0]$ $; E) \longrightarrow E$ are continuous; there exist nonnegative Lebesgue integrable functions $p_{i} \in L\left(J, R_{+}\right)(i=1,2)$ such that $\| K(t, s$, $x)\left\|\leq p_{1}(t)\right\| x \|_{C([-r, 0] ;)}$ and $\|H(t, s, x)\| \leq p_{2}(t)\|x\|_{C([-r, 0] ;)}$, for all $(t, s) \in D,(t, s) \in D_{0}, x \in C([-r, 0] ; E)$.

$\left(H_{3}\right) . \quad f: J \times C([-r, 0] ; E) \times C([-r, 0] ; E) \times C([-r, 0] ; E)$ $\longrightarrow E$ is continuous; there exist nonnegative Lebesgue integrable functions a, $L_{i} \in L\left(J, R_{+}\right)(i=1,2,3)$ such that $\| f(t$, $\left.x_{1}, x_{2}, x_{3}\right)\left\|\leq a(t)+\sum_{i=1}^{3} L_{i}(t)\right\| x_{i} \|_{C([-r, 0] ; E)}$, for all $t \in J, x_{i} \in C$ $([-r, 0] ; E)$.

Then, problem (4) has at least one mild solution $x \in C$ $\left(\left[-r, T_{0}\right] ; E\right)$.

Proof. Let us set the notation $R_{1}>0$ such that

$$
R_{1} \geq \frac{M^{\star} \phi_{0}+M^{\star} \int_{0}^{T_{0}} a(s) d s}{1-M^{\star}\left(\int_{0}^{T_{0}} L_{1}(s) d s+\int_{0}^{T_{0}} L_{2}(s) \int_{0}^{T_{0}} p_{1}(v) d v d s+\int_{0}^{T_{0}} L_{3}(s) \int_{0}^{T_{0}} p_{2}(v) d v d s\right)},
$$

where $\phi_{0}=\|\phi(0)\|$ and $\left(\int_{0}^{T_{0}} L_{1}(s) d s+\int_{0}^{T_{0}} L_{2}(s) \int_{0}^{T_{0}} p_{1}(v) d v\right.$ $\left.d s+\int_{0}^{T_{0}} L_{3}(s) \int_{0}^{T_{0}} p_{2}(v) d v d s\right)^{-1}>M^{\star}$.

First of all, we consider the set $B_{R_{1}}=\left\{x \in C\left(\left[-r, T_{0}\right] ; E\right)\right.$ : $\left.\|x\|_{C\left(\left[-r, T_{0}\right] ; E\right)} \leq R_{1}\right\}$ and show that $\Psi B_{R_{1}} \subset B_{R_{1}}$. By using conditions $\left(H_{2}\right)$ and $\left(H_{3}\right)$, for all $x \in B_{R_{1}}$, we have

$$
\begin{aligned}
\|(\Psi x)(t)\| \leq & \left\|U_{\beta}(t, 0) \phi(0)\right\|+\int_{0}^{t}\left\|U_{\beta}(t, s) f\left(s, x_{s}, \mathscr{K} x_{s}, \mathscr{H} x_{s}\right)\right\| d s \\
\leq & M^{\star} \phi_{0}+M^{\star} \int_{0}^{t}\left\|f\left(s, x_{s}, \mathscr{K} x_{s}, \mathscr{H} x_{s}\right)\right\| d s \leq M^{\star} \phi_{0} \\
& +M^{\star} \int_{0}^{t}\left(a(s)+L_{1}(s)\left\|x_{s}\right\|+L_{2}(s)\left\|\mathscr{K} x_{s}\right\|+L_{3}(s)\left\|\mathscr{H} x_{s}\right\|\right) d s \\
\leq & M^{\star} \phi_{0}+M^{\star} \int_{0}^{t} a(s) d s+M^{\star}\left(\int_{0}^{t} L_{1}(s) d s+\int_{0}^{t} L_{2}(s)\right. \\
& \left.\cdot \int_{0}^{s} p_{1}(v) d v d s+\int_{0}^{t} L_{3}(s) \int_{0}^{T_{0}} p_{2}(v) d v d s\right)\|x\|_{C([-r, 0] ; E)} \\
\leq & M^{\star} \phi_{0}+M^{\star} \int_{0}^{T_{0}} a(s) d s+M^{\star}\left(\int_{0}^{T_{0}} L_{1}(s) d s+\int_{0}^{T_{0}} L_{2}(s)\right. \\
& \left.\cdot \int_{0}^{T_{0}} p_{1}(v) d v d s+\int_{0}^{T_{0}} L_{3}(s) \int_{0}^{T_{0}} p_{2}(v) d v d s\right)\|x\|_{C\left(\left[-r, T_{0}\right]: E\right)} \leq R_{1} .
\end{aligned}
$$

So, we conclude that $\Psi$ maps $B_{R_{1}}$ into itself.

Second, we prove that $\Psi: B_{R_{1}} \longrightarrow B_{R_{1}}$ is continuous.

Let $\left\{x_{n}\right\}_{0}^{\infty} \subset C\left(\left[-r, T_{0}\right] ; E\right)$, with $x_{n} \longrightarrow x(n \longrightarrow \infty), x$ $\in C\left(\left[-r, T_{0}\right] ; E\right)$. Using the fact that $K: D \times C([-r, 0] ; E)$ $\longrightarrow E, H: D_{0} \times C([-r, 0] ; E) \longrightarrow E$, and $f: J \times C([-r, 0]$; $E) \times C([-r, 0] ; E) \times C([-r, 0] ; E) \longrightarrow E$ are continuous, we obtain

$f\left(t,\left(x_{n}\right)_{t}, \mathscr{K}\left(x_{n}\right)_{t}, \mathscr{H}\left(x_{n}\right)_{t}\right) \longrightarrow f\left(t, x_{t}, \mathscr{K} x_{t}, \mathscr{H} x_{t}\right)(n \longrightarrow \infty)$,

for any $t \in J$ uniformly. That is, for any $\varepsilon>0$, there exists a natural number $N_{0}$, for $n>N_{0}, t \in J$, such that

$$
\left\|f\left(t,\left(x_{n}\right)_{t}, \mathscr{K}\left(x_{n}\right)_{t}, \mathscr{H}\left(x_{n}\right)_{t}\right)-f\left(t, x_{t}, \mathscr{K} x_{t}, \mathscr{H} x_{t}\right)\right\| \leq \frac{\varepsilon}{M^{\star} T_{0}},
$$

which implies that

$$
\begin{aligned}
\left\|\left(\Psi x_{n}\right)(t)-(\Psi x)(t)\right\|= & \| \int_{0}^{t} U_{\beta}(t, s) f\left(s,\left(x_{n}\right)_{s}, \mathscr{K}\left(x_{n}\right)_{s}, \mathscr{H}\left(x_{n}\right)_{s}\right) d s \\
& -\int_{0}^{t} U_{\beta}(t, s) f\left(s, x_{s}, \mathscr{K} x_{s}, \mathscr{H} x_{s}\right) d s \| \\
\leq & M^{\star} \int_{0}^{t} \| f\left(s,\left(x_{n}\right)_{s}, \mathscr{K}\left(x_{n}\right)_{s}, \mathscr{H}\left(x_{n}\right)_{s}\right) \\
& -f\left(s, x_{s}, \mathscr{K} x_{s}, \mathscr{H} x_{s}\right) \| d s \leq M^{\star} T_{0} \frac{\varepsilon}{M^{\star} T_{0}}=\varepsilon .
\end{aligned}
$$

In consequence, $\Psi: B_{R_{1}} \longrightarrow B_{R_{1}}$ is continuous. Furthermore, we prove that $\Psi\left(B_{R_{1}}\right)$ is equicontinuous. 
To do this, let $L(s)=L_{1}(s)+L_{2}(s) \int_{0}^{T_{0}} p_{1}(v) d v+L_{3}(s) \int_{0}^{T_{0}}$ $p_{2}(v) d v$. Obviously, it is a nonnegative Lebesgue integrable function. For all $x \in B_{R_{1}}, t_{1}, t_{2} \in J\left(t_{1}<t_{2}\right)$, we have

$$
\begin{aligned}
& \left\|(\Psi x)\left(t_{2}\right)-(\Psi x)\left(t_{1}\right)\right\| \leq\left\|\left(U_{\beta}\left(t_{2}, 0\right)-U_{\beta}\left(t_{1}, 0\right)\right) \phi(0)\right\| \\
& +\left\|\int_{t_{1}}^{t_{2}} U_{\beta}\left(t_{2}, s\right) f\left(s, x_{s}, \mathscr{K} x_{s}, \mathscr{H} x_{s}\right) d s\right\| \\
& +\left\|\int_{0}^{t_{1}}\left(U_{\beta}\left(t_{2}, s\right)-U_{\beta}\left(t_{1}, s\right)\right) f\left(s, x_{s}, \mathscr{K} x_{s}, \mathscr{H} x_{s}\right) d s\right\| \\
& \leq \phi_{0}\left\|U_{\beta}\left(t_{2}, 0\right)-U_{\beta}\left(t_{1}, 0\right)\right\|+M^{\star} \int_{t_{1}}^{t_{2}}\left\|f\left(s, x_{s}, \mathscr{K} x_{s}, \mathscr{H} x_{s}\right)\right\| d s \\
& \quad+\sup _{s \in J}\left\|U_{\beta}\left(t_{2}, s\right)-U_{\beta}\left(t_{1}, s\right)\right\| \int_{0}^{t_{1}}\left\|f\left(s, x_{s}, \mathscr{K} x_{s}, \mathscr{H} x_{s}\right)\right\| d s \\
& \leq \phi_{0}\left\|U_{\beta}\left(t_{2}, 0\right)-U_{\beta}\left(t_{1}, 0\right)\right\|+M^{\star} \int_{t_{1}}^{t_{2}}\left(a(s)+L(s) R_{1}\right) d s \\
& \quad+\sup _{s \in J}\left\|U_{\beta}\left(t_{2}, s\right)-U_{\beta}\left(t_{1}, s\right)\right\| \int_{0}^{t_{1}}\left(a(s)+L(s) R_{1}\right) d s \\
& =: I_{1}+I_{2}+I_{3} .
\end{aligned}
$$

In view of condition $\left(H_{1}\right)$, compactness of the resolvent operator $U_{\beta}(t, s)(t, s)>0$ implies the continuity in the uniform operator topology. That is, for any $\varepsilon>0$, there exists $\delta_{1}>0$, for any $\left|t_{2}-t_{1}\right|<\delta_{1}, t_{1}, t_{2} \in J$, such that $I_{3}<\varepsilon / 3$. Hence, for the above $\varepsilon>0$, by using properties of $U_{\beta}(t, s)$ and the above inequalities, there exists $\delta>0\left(\delta<\delta_{1}\right)$ such that $\left\|(\Psi x)\left(t_{2}\right)-(\Psi x)\left(t_{1}\right)\right\|<\varepsilon$, for any $\left|t_{2}-t_{1}\right|<\delta, t_{1}, t_{2} \in J$ . Consequently, $\Psi\left(B_{R_{1}}\right)$ is equicontinuous.

In the end, we prove that $\Psi\left(B_{R_{1}}\right)$ is precompact.

For any fixed $t\left(t \in\left[-r, T_{0}\right]\right)$ and $0<\varepsilon<t$, the operator $\left(\Psi_{\varepsilon} x\right)(t)$ is defined by

$$
\left(\Psi_{\varepsilon} x\right)(t)=\left\{\begin{array}{l}
U_{\beta}(t, 0) \phi(0)+\int_{0}^{t-\varepsilon} U_{\beta}(t, s) f\left(s, x_{s}, \mathscr{K} x_{s}, \mathscr{H} x_{s}\right) d s, \quad t \in\left[0, T_{0}\right], \\
\phi(t), \quad t \in[-r, 0] .
\end{array}\right.
$$

Since $U_{\beta}(t, s)(t, s)>0$ is a compact resolvent operator, then the set $Y_{\varepsilon}(t)=\left\{\left(\Psi_{\varepsilon} x\right)(t): x \in B_{R_{1}}\right\}$ is relatively compact in $E$ for any $\varepsilon(0<\varepsilon<t)$.

Moreover, for any $x \in B_{R_{1}}$, one can find that

$$
\begin{aligned}
\left\|(\Psi x)(t)-\left(\Psi_{\varepsilon} x\right)(t)\right\| & =\left\|\int_{t-\varepsilon}^{t} U_{\beta}(t, s) f\left(s, x_{s}, \mathscr{K} x_{s}, \mathscr{H} x_{s}\right) d s\right\| \\
& \leq M^{\star}\left\|\int_{t-\varepsilon}^{t} f\left(s, x_{s}, \mathscr{K} x_{s}, \mathscr{H} x_{s}\right) d s\right\| \\
& \leq M^{\star} \int_{t-\varepsilon}^{t}\left(a(s)+L(s) R_{1}\right) d s \\
& \leq M^{\star}\left(\|a(s)\|+\|L(s)\| R_{1}\right) \varepsilon .
\end{aligned}
$$

Thus, $Y(t)=\left\{(\Psi x)(t): x \in B_{R_{1}}\right\}$ is totally bounded. Hence, $Y(t)$ is relatively compact in $E$, and so, based on the ArzelàAscoli theorem, $\Psi: B_{\mathrm{R}_{1}} \longrightarrow B_{R_{1}}$ is completely continuous. As all the assumptions of the Schauder fixed point theorem are satisfied, the conclusion implies that the operator $\Psi$ has a fixed point $x$ in $C\left(\left[-r ; T_{0}\right], E\right)$, which is a global mild solution of problem (4). This completes the proof.

Next, we develop the existence of global mild solutions for problem (4) via the measure of noncompactness and fixed point theorem. Furthermore, we employ the notations: $T_{R}=\left\{x \in C\left(\left[-r, T_{0}\right] ; E\right):\|x\|_{C\left(\left[-r, T_{0}\right] ; E\right)} \leq R\right\}, k_{0}=\sup$ $\left\{\left\|K\left(t, s, x_{s}\right)\right\|:\left(t, s, x_{s}\right) \in D \times C([-r, 0] ; E)\right\}, h_{0}=\sup \{\| H(t$, $\left.\left.s, x_{s}\right) \|:\left(t, s, x_{s}\right) \in D_{0} \times C([-r, 0] ; E)\right\}$, and $R \geq \max \left\{T_{0} k_{0}\right.$, $\left.T_{0} h_{0}\right\}$.

Theorem 13. Assume that $\left(H_{1}\right)$ and the following conditions hold:

$\left(H_{4}\right)$. The function $f: J \times T_{R} \times T_{R} \times T_{R} \longrightarrow E$ is bounded and continuous, which satisfies

$$
\lim _{R \rightarrow \infty} \sup \frac{M(R)}{R}<\frac{1}{T_{0} M^{\star}}
$$

where $M(R)=\sup \left\{\left\|f\left(t, x_{1}, x_{2}, x_{3}\right)\right\|:\left(t, x_{1}, x_{2}, x_{3}\right) \in J \times T_{R}\right.$ $\left.\times T_{R} \times T_{R}\right\}$.

$\left(\mathrm{H}_{5}\right)$. For any $\mathrm{R}$, there exist nonnegative Lebesgue integrable functions $q_{i} \in L\left(J, R_{+}\right),(i=1,2,3,4,5)$ such that for any equicontinuous and countable set $D_{i} \subset T_{R}(i=1,2,3), \alpha(f(t$, $\left.\left.D_{1}, D_{2}, D_{3}\right)\right) \leq \sum_{i=1}^{3} q_{i}(t) \alpha\left(D_{i}\right), \quad \alpha\left(K\left(t, s, D_{2}\right)\right) \leq q_{4}(t) \alpha\left(D_{2}\right)$, and $\alpha\left(H\left(t, s, D_{3}\right)\right) \leq q_{5}(t) \alpha\left(D_{3}\right)$.

$\left(H_{6}\right) . \quad 2 M^{\star} \int_{0}^{T_{0}}\left(q_{1}(s)+q_{2}(s) \int_{0}^{T_{0}} q_{4}(v) d v+q_{3}(s) \int_{0}^{T_{0}} q_{5}(v) d\right.$ $v) d s<1$.

Then, problem (4) has at least one mild solution.

Proof. By $\left(H_{4}\right)$, there exists $0<\mu<1 / T_{0} M^{\star}$ and $R_{0}>0$, for any $R \geq R_{0}$, such that

$$
M(R)<\mu R
$$

Let $R^{\star}=\max \left\{R_{0}, M^{\star} \phi_{0}\left(1-M^{\star} T_{0} \mu\right)^{-1}\right\}$; we first consider the set $B_{R^{\star}}=\left\{x \in C\left(\left[-r, T_{0}\right] ; E\right):\|x\|_{C\left(\left[-r, T_{0}\right] ; E\right)} \leq R^{\star}\right\}$ and show that $\Psi B_{R^{\star}} \subset B_{R^{\star}}$. From the above inequality, for all $x \in B_{R^{\star}}$, we have

$$
\begin{aligned}
\|\Psi x\|_{C\left(\left[-r, T_{0}\right] ; E\right)} & \leq\left\|U_{\beta}(t, 0) \phi(0)\right\|+\int_{0}^{t}\left\|U_{\beta}(t, s)\right\|\left\|f\left(s, x_{s}, \mathscr{K} x_{s}, \mathscr{H} x_{s}\right)\right\| d s \\
& \leq M^{\star} \phi_{0}+M^{\star} T_{0} M\left(R^{\star}\right) \leq M^{\star} \phi_{0}+M^{\star} T_{0} \mu R^{\star} \leq R^{\star} .
\end{aligned}
$$

Meanwhile, applying the arguments employed in the proof of Theorem 12, we conclude that $\Psi$ is a continuous and bounded operator on $B_{R^{\star}}$. 
Then, we prove that $\Psi\left(B_{R^{\star}}\right)$ is equicontinuous. For any $x \in B_{R^{\star}}, t_{1}, t_{2} \in J\left(t_{1}<t_{2}\right)$, we have

$$
\begin{aligned}
& \left\|(\Psi x)\left(t_{2}\right)-(\Psi x)\left(t_{1}\right)\right\| \leq\left\|\left(U_{\beta}\left(t_{2}, 0\right)-U_{\beta}\left(t_{1}, 0\right)\right) \phi(0)\right\| \\
& \quad+\int_{t_{1}}^{t_{2}}\left\|U_{\beta}\left(t_{2}, s\right) f\left(s, x_{s}, \mathscr{K} x_{s}, \mathscr{H} x_{s}\right)\right\| d s \\
& \quad+\int_{0}^{t_{1}}\left\|\left(U_{\beta}\left(t_{2}, s\right)-U_{\beta}\left(t_{1}, s\right)\right) f\left(s, x_{s}, \mathscr{K} x_{s}, \mathscr{H} x_{s}\right)\right\| d s \\
& \leq \phi_{0}\left\|U_{\beta}\left(t_{2}, 0\right)-U_{\beta}\left(t_{1}, 0\right)\right\|+M^{\star}\left(t_{2}-t_{1}\right) M\left(R^{\star}\right) \\
& \quad+\sup _{s \in J}\left\|U_{\beta}\left(t_{2}, s\right)-U_{\beta}\left(t_{1}, s\right)\right\| M\left(R^{\star}\right) t_{1} .
\end{aligned}
$$

By $\left(H_{1}\right)$, the compactness of $U_{\beta}(t, s)$, for $(t, s)>0$, implies the continuity in the uniform operator topology. Namely, for any $\varepsilon>0$, there exists $\delta_{1}>0$, for any $\left|t_{2}-t_{1}\right|<$ $\delta_{1}, t_{1}, t_{2} \in J$, such that

$$
\sup _{s \in J}\left\|U_{\beta}\left(t_{2}, s\right)-U_{\beta}\left(t_{1}, s\right)\right\| M\left(R^{\star}\right) t_{1}<\frac{\varepsilon}{3} .
$$

Therefore, for the above $\varepsilon>0$, there exists $\delta>0\left(\delta<\delta_{1}\right)$ such that $\left\|(\Psi x)\left(t_{2}\right)-(\Psi x)\left(t_{1}\right)\right\|<\varepsilon$, for all $x \in B_{R^{\star}},\left|t_{2}-t_{1}\right|$ $<\delta, t_{1}, t_{2} \in J$, which shows that $\Psi\left(B_{R^{*}}\right)$ is equicontinuous. In view of Lemma $7, \bar{C} o \Psi\left(B_{R^{\star}}\right) \subset B_{R^{\star}}$ is bounded and equicontinuous.

Finally, we prove that $\Psi: \bar{C} o \Psi\left(B_{R^{*}}\right) \longrightarrow \bar{C} o \Psi\left(B_{R^{*}}\right)$ is a condensing operator. By Lemma 9, for any $D \subset \bar{C} o \Psi\left(B_{R^{\star}}\right)$, there exists a countable set $D_{0}=\left\{x_{n}\right\} \subset D$ such that

$$
\alpha(\Psi(D)) \leq 2 \alpha\left(\Psi\left(D_{0}\right)\right)
$$

By using condition $\left(H_{5}\right)$ and Lemma 8 , we obtain

$$
\begin{aligned}
\alpha\left(\Psi\left(D_{0}\right)(t)\right)= & \alpha\left(\int_{0}^{t} U_{\beta}(t, s) f\left(s,\left(D_{0}\right)_{s}, \mathscr{K}\left(D_{0}\right)_{s}, \mathscr{H}\left(D_{0}\right)_{s}\right) d s\right) \\
\leq & M^{\star} \int_{0}^{t} \alpha\left(f\left(s,\left(D_{0}\right)_{s}, \mathscr{K}\left(D_{0}\right)_{s}, \mathscr{H}\left(D_{0}\right)_{s}\right)\right) d s \\
\leq & M^{\star} \int_{0}^{t}\left(q_{1}(s) \alpha\left(\left(D_{0}\right)_{s}\right)+q_{2}(s) \alpha\left(\mathscr{K}\left(D_{0}\right)_{s}\right)\right. \\
& \left.+q_{3}(s) \alpha\left(\mathscr{H}\left(D_{0}\right)_{s}\right)\right) d s \\
\leq & M^{\star} \int_{0}^{t}\left(q_{1}(s)+q_{2}(s) \int_{0}^{s} q_{4}(v) d(v)\right. \\
& \left.+q_{3}(s) \int_{0}^{T_{0}} q_{5}(v) d(v)\right) d s \alpha(D) .
\end{aligned}
$$

In addition, using Lemma 8 , we have

$$
\alpha\left(\Psi\left(D_{0}\right)\right)=\max _{t \in J} \alpha\left(\Psi\left(D_{0}\right)(t)\right)
$$

Consequently,

$$
\begin{gathered}
\alpha(\Psi(D)) \leq 2 M^{\star} \int_{0}^{T_{0}}\left(q_{1}(s)+q_{2}(s) \int_{0}^{T_{0}} q_{4}(v) d(v)\right. \\
\left.+q_{3}(s) \int_{0}^{T_{0}} q_{5}(v) d(v)\right) d s \alpha(D) .
\end{gathered}
$$

By $\left(H_{6}\right)$, we obtain that $\Psi$ is a condensing operator on $\bar{C} o \Psi\left(B_{R^{*}}\right)$. By Lemma 10, there exists at least one fixed point $x \in \bar{C} o \Psi\left(B_{R^{*}}\right) \subset C\left(\left[-r, T_{0}\right] ; E\right)$ for $\Psi$. In conclusion, problem (4) has at least one global mild solution. This completes the proof.

Remark 14. Theorems 12 and 13 above are concluded under the conditions that $U_{\beta}(t, s)$ is compact for $t, s>0$ and the functions $f, K$, and $H$ satisfy corresponding conditions; in contrast, when the resolvent operator $\mathrm{U}_{\beta}(t, s)$ is noncompact, we could obtain Theorem 15 if $f, K$, and $H$ meet the Lipschitz conditions.

Theorem 15. Assume that the following conditions hold:

$\left(H_{7}\right) . \quad f: J \times C([-r, 0] ; E) \times C([-r, 0] ; E) \times C([-r, 0] ; E)$ $\longrightarrow E$ is continuous; there exist nonnegative Lebesgue integrable functions $g_{i} \in L\left(J, R_{+}\right)(i=1,2,3)$, for all $t \in J, u_{i}, v_{i} \in$ E, such that

$\left\|f\left(t, u_{1}, u_{2}, u_{3}\right)-f\left(t, v_{1}, v_{2}, v_{3}\right)\right\| \leq \sum_{i=1}^{3} g_{i}(t)\left\|u_{i}-v_{i}\right\|_{C([-r, 0] ; E)}$.

$\left(H_{8}\right) . K: D \times C([-r, 0] ; E) \longrightarrow E$ and $H: D_{0} \times C([-r, 0]$ $; E) \longrightarrow E$; there exist nonnegative Lebesgue integrable functions $g_{4}, g_{5} \in L\left(J, R_{+}\right)$, for all $u, v \in E,(t, s) \in D,(t, s) \in D_{0}$ such that

$$
\begin{aligned}
& \|K(t, s, u)-K(t, s, v)\| \leq g_{4}(t)\|u-v\|_{C([-r, 0] ; E)}, \\
& \|H(t, s, u)-H(t, s, v)\| \leq g_{5}(t)\|u-v\|_{C([-r, 0] ; E)} . \\
& \left(H_{9}\right) . \quad M^{\star} \int_{0}^{T_{0}}\left(g_{1}(s)+g_{2}(s) \int_{0}^{T_{0}} g_{4}(v) d v+g_{3}(s) \int_{0}^{T_{0}} g_{5}(v) d\right.
\end{aligned}
$$
$v) d s<1$.

Then, problem (4) has a unique mild solution.

Proof. For any $u, v \in C\left(\left[-r, T_{0}\right] ; E\right)$,

$$
\begin{aligned}
& \|(\Psi u)(t)-(\Psi v)(t)\| \leq M^{\star} \int_{0}^{t} \| f\left(s, u_{s}, \mathscr{K} u_{s}, \mathscr{H} u_{s}\right) \\
& \quad-f\left(s, v_{s}, \mathscr{K} v_{s}, \mathscr{H} v_{s}\right) \| d s \leq M^{\star} \int_{0}^{t}\left(g_{1}(s)\left\|u_{s}-v_{s}\right\|\right. \\
& \left.\quad+g_{2}(s)\left\|\mathscr{K} u_{s}-\mathscr{K} v_{s}\right\|+g_{3}(s)\left\|\mathscr{H} u_{s}-\mathscr{H} v_{s}\right\|\right) d s \leq M^{\star} \int_{0}^{T_{0}} \\
& \quad \cdot\left(g_{1}(s)+g_{2}(s) \int_{0}^{T_{0}} g_{4}(v) d v+g_{3}(s) \int_{0}^{T_{0}} g_{5}(v) d v\right) d s\|u-v\|_{C\left(\left[-r, T_{0}\right] ; E\right)} .
\end{aligned}
$$

By $\left(H_{9}\right)$, we have $\|\Psi u-\Psi v\|_{C\left(\left[-r, T_{0}\right] ; E\right)}<\|u-v\|_{C\left(\left[-r, T_{0}\right] ; E\right)}$. 
These arguments enable us to conclude that the operator $\Psi$ is a contraction mapping. Hence, the operator $\Psi$ has a unique fixed point $x^{\star} \in C\left(\left[-r, T_{0}\right] ; E\right)$, which implies that problem (4) has a unique global mild solution. This completes the proof.

Remark 16. In Theorem 15, we develop the uniqueness of the mild solution for problem (4) via the Banach contraction mapping principle. In conditions $\left(H_{7}\right)$ and $\left(H_{8}\right), g_{i} \in L(J$, $\left.R_{+}\right)(i=1,2,3,4,5)$ turn out to be nonnegative Lebesgue integrable functions instead of constants.

\section{An Application}

In order to show the application of the main results, we consider the following problem:

$$
\left\{\begin{array}{l}
{ }^{c} D_{t}^{\beta} x(z, t)=t^{2} \frac{\partial^{2}}{\partial z^{2}} x(z, t)+J_{t}^{1-\beta}\left(\frac{t}{1+t^{2}} x(z, t+\theta)+\frac{1}{1+t^{2}} \int_{0}^{t} a(s) x(z, s+\theta) d s+\frac{1}{1+e^{t}} \int_{0}^{1} b(s) x(z, s+\theta) d s\right) \\
0<t \leq 1, \quad z \in \Omega, \theta \in[-r, 0] \\
x(z, \theta)=\varphi(z, \theta), \quad z \in \Omega, \theta \in[-r, 0]
\end{array}\right.
$$

where $0<\beta<1,{ }^{c} D_{t}^{\beta}$ is the Caputo fractional derivative of order $\beta, J_{t}^{1-\beta}$ is the Riemann-Liouville fractional integral of order $1-\beta, \Omega \subset \mathbb{R}^{n}$ is a bounded domain with regular boundary $\partial \Omega$, and $\varphi \in C([-r, 0] ; E), E=C(\bar{\Omega} ; \mathbb{R}), \bar{\Omega}=\Omega \bigcup$ $\partial \Omega$.

By setting $x(t)=x(\cdot, t)$, problem (33) can be rewritten as the following abstract form:

$$
\left\{\begin{array}{l}
{ }^{c} D_{t}^{\beta} x(t)=A(t) x(t)+J_{t}^{1-\beta} f\left(t, x_{t}, \mathscr{K} x_{t}, \mathscr{H} x_{t}\right), \quad t \in[0,1], \\
x(t)=\varphi(t), \quad t \in[-r, 0],
\end{array}\right.
$$

where $x_{t}=x(t+\theta), f\left(t, x_{t}, \mathscr{K} x_{t}, \mathscr{H} x_{t}\right)=\left(\left(t /\left(1+t^{2}\right)\right) x_{t}+(1 /\right.$ $\left.\left.\left(1+t^{2}\right)\right) \int_{0}^{t} a(s) x_{s} d s+\left(1 /\left(1+e^{t}\right)\right) \int_{0}^{1} b(s) x_{s} d s\right)$, and

$$
\left\{\begin{array}{l}
D(A)=\left\{x \in C(\bar{\Omega}, R): x^{\prime \prime} \in C(\bar{\Omega}, R)\right\}, \\
A(t) x=x^{\prime \prime}, \quad t \in[-r, 0] .
\end{array}\right.
$$

It is well known that the operator $A(t)$ generates a $\beta$ -resolvent family $U_{\beta}(t, s)[23,25]$. Let equation (34) satisfy the conditions of Theorems 12-15; then, problem (34) has a global mild solution, which means that problem (33) has a mild solution.

\section{Conclusion}

In this paper, we study the existence and uniqueness of the global mild solutions for the fractional integrodifferential equations of mixed type with delay. Under the condition of the compact resolvent operator, we obtain Theorems 12 and 13, respectively, via various fixed point theorems and the measure of noncompactness. Theorem 15 is established by using the Banach contraction mapping principle under the condition of the noncompact resolvent operator. Furthermore, an example is provided to illustrate the main theorems.
The kernels $K$ and $H$ of the operators $\mathscr{K}$ and $\mathscr{H}$ are nonlinear functions; meanwhile, the operator $A(t)$ is dependent on $t$. As a consequence, our main theorems improve and generalize many corresponding results by using different methods.

\section{Data Availability}

No data were used to support this study.

\section{Conflicts of Interest}

The authors declare that they have no conflicts of interest.

\section{Authors' Contributions}

All authors contributed equally and significantly in writing this article. All authors read and approved the final manuscript.

\section{Acknowledgments}

This research is supported by the National Science Foundation of China (Grant No. 11971264), the National Key R\&D Program of China (Grant No. 2018YFA0703900), the National Natural Science Foundation of China (No. 62073190), and the Project of Shandong Province Higher Educational Science and Technology Program (No. J16LI14).

\section{References}

[1] P. Chen, X. Zhang, and Y. Li, "Existence and approximate controllability of fractional evolution equations with nonlocal conditions via resolvent operators," Fractional Calculus and Applied Analysis, vol. 23, no. 1, pp. 268-291, 2020.

[2] R. P. Agarwal, D. Baleanu, J. J. Nieto, D. F. M. Torres, and Y. Zhou, "A survey on fuzzy fractional differential and optimal control nonlocal evolution equations," Journal of Computational and Applied Mathematics, vol. 339, pp. 3-29, 2018. 
[3] P. Chen, X. Zhang, and Y. Li, "Cauchy problem for fractional non-autonomous evolution equations," Banach Journal of Mathematical Analysis, vol. 14, no. 2, pp. 559-584, 2020.

[4] B. Zhu, L. Liu, and Y. Wu, "Existence and uniqueness of global mild solutions for a class of nonlinear fractional reactiondiffusion equations with delay," Computers and Mathematics with Applications, vol. 78, no. 6, pp. 1811-1818, 2019.

[5] P. Chen, X. Zhang, and Y. Li, "Study on fractional nonautonomous evolution equations with delay," Computers and Mathematics with Applications, vol. 73, no. 5, pp. 794-803, 2017.

[6] A. Kilbas, H. Srivastava, and J. Trujillo, Theory and Applications of Fractional Differential Equations, North-Holland Mathematics Studies, vol. 204, Elsevier Science B.V, Amsterdam, 2006.

[7] E. Alvarez-Pardo and C. Lizama, "Weighted pseudo almost automorphic mild solutions for two-term fractional order differential equations," Applied Mathematics and Computation, vol. 271, pp. 154-167, 2015.

[8] H. Li and Y. Kao, "Mittag-Leffler stability for a new coupled system of fractional-order differential equations with impulses," Applied Mathematics and Computation, vol. 361, pp. 22-31, 2019.

[9] P. Chen, X. Zhang, and Y. Li, "Fractional non-autonomous evolution equation with nonlocal conditions," Journal of Pseudo-Differential Operators and Applications, vol. 10, no. 4, pp. 955-973, 2019.

[10] B. Zhu and B. Han, "Existence and uniqueness of mild solutions for fractional partial integro-differential equations," Mediterranean Journal of Mathematics, vol. 17, no. 4, 2020.

[11] A. Chadha and D. N. Pandey, "Existence results for an impulsive neutral stochastic fractional integro- differential equation with infinite delay," Nonlinear Analysis, vol. 128, pp. 149-175, 2015.

[12] P. Chen and Y. Li, "Nonlocal Cauchy problem for fractional stochastic evolution equations in Hilbert spaces," Collectanea Mathematica, vol. 66, no. 1, pp. 63-76, 2015.

[13] P. Chen, Y. Li, and H. Yang, "Perturbation method for nonlocal impulsive evolution equations," Nonlinear Analysis, vol. 8, pp. 22-30, 2013.

[14] P. Chen, X. Zhang, and Y. Li, “Approximate controllability of non-autonomous evolution system with nonlocal conditions," Journal of Dynamical and Control Systems, vol. 26, no. 1, pp. 1-16, 2020.

[15] P. Chen, X. Zhang, and Y. Li, "Non-autonomous parabolic evolution equations with non-instantaneous impulses governed by noncompact evolution families," Journal of Fixed Point Theory and Applications, vol. 21, no. 3, 2019.

[16] P. Chen, X. Zhang, and Y. Li, "Non-autonomous evolution equations of parabolic type with non-instantaneous impulses," Mediterranean Journal of Mathematics, vol. 16, no. 5, 2019.

[17] P. Chen, Department of Mathematics, Northwest Normal University, Lanzhou 730070, China, Y. Li, and X. Zhang, "Cauchy problem for stochastic non-autonomous evolution equations governed by noncompact evolution families," Discrete and Continuous Dynamical Systems Series B, vol. 26, no. 3, pp. 1531-1547, 2021.

[18] B. Zhu, L. Liu, and Y. Wu, "Local and global existence of mild solutions for a class of nonlinear fractional reaction-diffusion equations with delay," Applied Mathematics Letters, vol. 61, pp. 73-79, 2016.
[19] H. Gou and B. Li, "Local and global existence of mild solution to impulsive fractional semilinear integro-differential equation with noncompact semigroup," Communications in Nonlinear Science and Numerical Simulation, vol. 42, pp. 204-214, 2017.

[20] Z. Ouyang, "Existence and uniqueness of the solutions for a class of nonlinear fractional order partial differential equations with delay," Computers and Mathematics with Applications, vol. 61, no. 4, pp. 860-870, 2011.

[21] L. Liu, F. Guo, C. Wu, and Y. Wu, "Existence theorems of global solutions for nonlinear Volterra type integral equations in Banach spaces," Journal of Mathematical Analysis and Applications, vol. 309, no. 2, pp. 638-649, 2005.

[22] P. Chen, Department of Mathematics, Northwest Normal University, Lanzhou 730070, China, X. Zhang, and Y. Li, “A blowup alternative result for fractional non-autonomous evolution equation of Volterra type," Communications on Pure and Applied Analysis, vol. 17, no. 5, pp. 1975-1992, 2018.

[23] B. Zhu, B. Han, and L. Liu, "Existence of mild solutions for a class of fractional semilinear integro-diffusion equation of mixed type," Acta Mathematica Scientia, vol. 39, pp. 1334$1341,2019$.

[24] L. Liu, "Iterative method for solutions and coupled quasisolutions of nonlinear integro-differential equations of mixed type in Banach spaces," Nonlinear Analysis, vol. 42, no. 4, pp. 583-598, 2000.

[25] K. Li and J. Jia, "Existence and uniqueness of mild solutions for abstract delay fractional differential equations," Computers Mathematics with Applications, vol. 62, no. 3, pp. 1398-1404, 2011.

[26] S. Samko, A. Kilbas, and O. Marichev, Fractional Integrals and Derivatives: Theory and Applications, Gordon and Breach, Amsterdam, 1993.

[27] M. Caputo, "Linear models of dissipation whose Q is almost frequency independent-II," Journal of the Royal Astronomical Society, vol. 13, no. 5, pp. 529-539, 1967.

[28] D. Araya and C. Lizama, "Almost automorphic mild solutions to fractional differential equations," Nonlinear Analysis, vol. 69, no. 11, pp. 3692-3705, 2008.

[29] A. Debbouche and D. Baleanu, "Controllability of fractional evolution nonlocal impulsive quasilinear delay integrodifferential systems," Computers and Mathematics with Applications, vol. 62, no. 3, pp. 1442-1450, 2011.

[30] C. Lizama, A. Pereira, and R. Ponce, "On the compactness of fractional resolvent operator functions," Semigroup Forum, vol. 93, no. 2, pp. 363-374, 2016.

[31] D. Deimling, Nonlinear Functional Analysis, Springer, Berlin, 1985. 